\title{
CRUD On The Web - Pedagogical Modules For Web-Based Data Access
}

Kelly Fadel, Utah State University, USA

David Olsen, Utah State University, USA

Karina Hauser, Utah State University, USA

\begin{abstract}
The growing popularity of Internet-enabled commerce has produced increased demand for Information Technology (IT) professionals who are skilled in the development and management of data-driven, Web-based business applications. Many academic programs in information systems offer courses on relational database design and management, as well as courses on Web development using technologies such as PHP or Microsoft's ASP.NET. However, such courses typically contain independent content, which tends to leave students with a fragmented understanding of how these technologies (i.e. the Web and relational databases) interact. In this paper, we present integrated instructional modules for teaching best practices in connecting advanced Web applications with a relational database backend. The objective of these modules is to provide students with a seamless context for developing both a relational database and a Web interface supporting database transactions.
\end{abstract}

\section{INTRODUCTION}

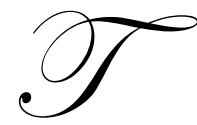

he growing popularity of Internet-enabled commerce has produced increased demand for Information Technology (IT) professionals who are skilled in the development and management of data-driven, Web-based business applications. A 2005 study investigating highly sought-after IT skills reported that "not only has Web programming jumped into a commanding lead in the total number of jobs requiring programming skills, it is now mentioned in an impressive $42.6 \%$ of job ads" [8, p. 91] More, recently, Litecky, et al. [5], report that Web programming topped the list of the most frequently demanded skills by employers, appearing in $26.5 \%$ of the reviewed job postings. Not far behind is the market for database programming and management skills: both studies ranked SQL programming within the top 10 most demanded skills in the IT job market.

The obvious popularity of Web and SQL programming skills highlights the need for academic IS programs to enable emerging IT professionals to meet this demand. Many academic programs in information systems offer courses on relational database design and management, as well as courses on Web development using technologies such as PHP or Microsoft's ASP.NET [4]. However, such courses typically contain independent content, which tends to leave students with a fragmented understanding of how these technologies (i.e. the Web and relational databases) interact. Because today's Web applications rely increasingly on dynamically updated data from a data source such as a relational database, Web application developers must have a working knowledge of both the backend database and the front-end Web interface. Consequently, there is a need for comprehensive pedagogical material that provides an integrated context for teaching these skills.

To address this need, we have developed integrated instructional modules for teaching best practices in connecting advanced Web applications with a relational database backend. These modules introduce students to the common practices of connecting Web applications to a relational database and performing CRUD (Create, Read/Retrieve, Update, Delete/Destroy) operations on the data through a Web-based interface. These modules were successfully integrated in a Management Information Systems (MIS) undergraduate program that offers both a database management course and a Web development course. Students are first introduced to the database modules in the database management course, after which they work though the Web modules in the Web development 
course. The objective of these integrated modules is to provide students with a seamless context for developing both a relational database and a Web interface supporting database transactions.

The next section presents the learning modules we developed. Database modules are first presented, followed by the Web Modules. The technology used in teaching these modules is Microsoft SQL Server 2005 and ASP.NET 2.0. However, implementation of the concepts presented can be modified to suit any similar technological platform.

\section{CONTEXT DESCRIPTION}

The context for this case is a United States-based foreign currency exchange service. The database contains account and contact information about each customer of the service. Customers can be individual persons or corporations, and each can execute transactions in which they exchange US Dollars for other currencies. The database also contains utility/lookup tables for information such as US States and a calendar identifying business days, holidays, and weekends.

\section{DATABASE MODULES}

This section first describes the conceptual data model followed by the Data Definition Language (DDL) required to create the database structure.

\section{Conceptual Data Model}

Since the introduction of Codd's [3] relational model, conceptual data modeling has become an integral part of database development. The purpose of conceptual data models is to gather and describe the information involve in the business process at a high-level independent of the DBMS used later in the implementation. It is an important step in the requirements analysis phase and helps to facilitate communication between end-users, database administrators and developers. Conceptual data modeling should be an integral part of any database class, but an analysis of textbooks [6] shows that even textbook authors have problems creating integrated and consistent examples for instructors and students to follow. The entity-relationship (ER) diagram developed by Chen [2] is the most widely used diagram used to represent data. The ER Diagram for the current case is shown in Figure 1 below $^{12}$ :

\footnotetext{
${ }^{1}$ Attributes for each entity are omitted from the ER Diagram for the sake of clarity. Attributes are identified in the data definition language presented in the following section.

2 The Calendar entity is included as a utility for easily identifying work days, holidays, etc. Relationships could be drawn between the calendar entity and other entities that have date attributes (e.g. CustAddresses). However, for the sake of clarity, the Calendar entity is shown relating only to the Transactions entity.
} 


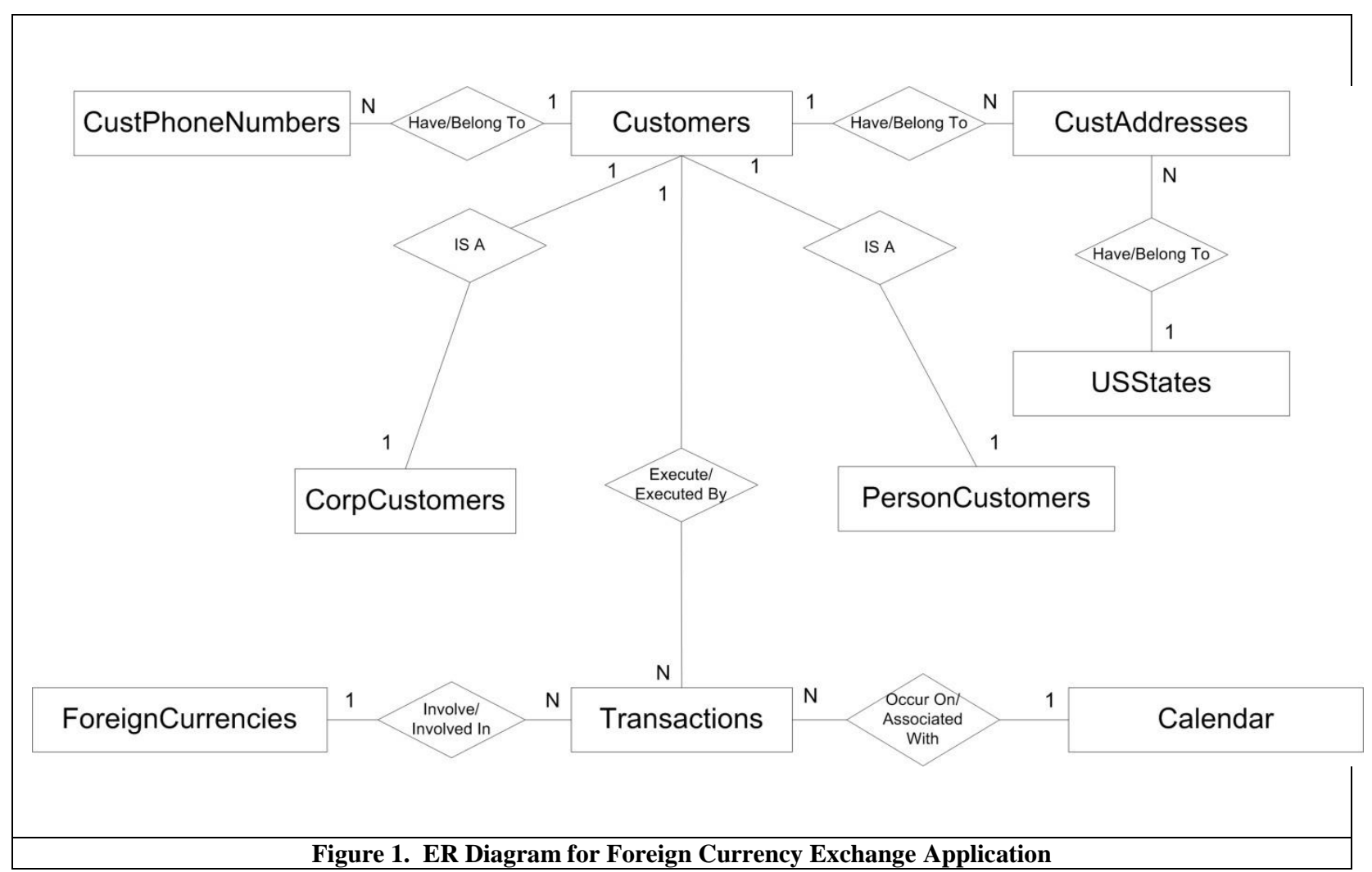

\title{
Data Definition Language For Creating And Populating Database
}

We now present the DDL for creating the database structures (tables, constraints, triggers, etc.) that implement the conceptual data model. This example is written for the Microsoft SQL Server 2005 DBMS; however, the SQL code is ANSI-compliant and should therefore work in any DBMS environment.

\section{Listing 1. DDL for creating Database Tables and Constraints}

\section{CREATE TABLE Calendar}

(

$\begin{array}{llll}\text { ActualDate } & \text { DATETIME } & \text { NOT NULL } & \text { PRIMARY KEY, } \\ \text { MonthName } & \text { CHAR(15) } & \text { NULL, } & \\ \text { DayNumber } & \text { INT } & \text { NULL, } \\ \text { YearNumber } & \text { INT } & \text { NULL, } \\ \text { DayOfWeek } & \text { CHAR(15) } & \text { NULL }\end{array}$

CHECK (DayOfWeek IN ('Sunday','Monday','Tuesday',

'Wednesday', 'Thursday', 'Friday', 'Saturday')),
DayType CHAR(15) NULL
CHECK (DayType IN ('Business','Weekend', 'Holiday')),

)

CREATE TABLE USStates

(
Abbreviation
char (2)
NOT NULL
PRIMARY KEY,

)

\begin{abstract}
StateName
\end{abstract}
char (25)

NOT NULL 


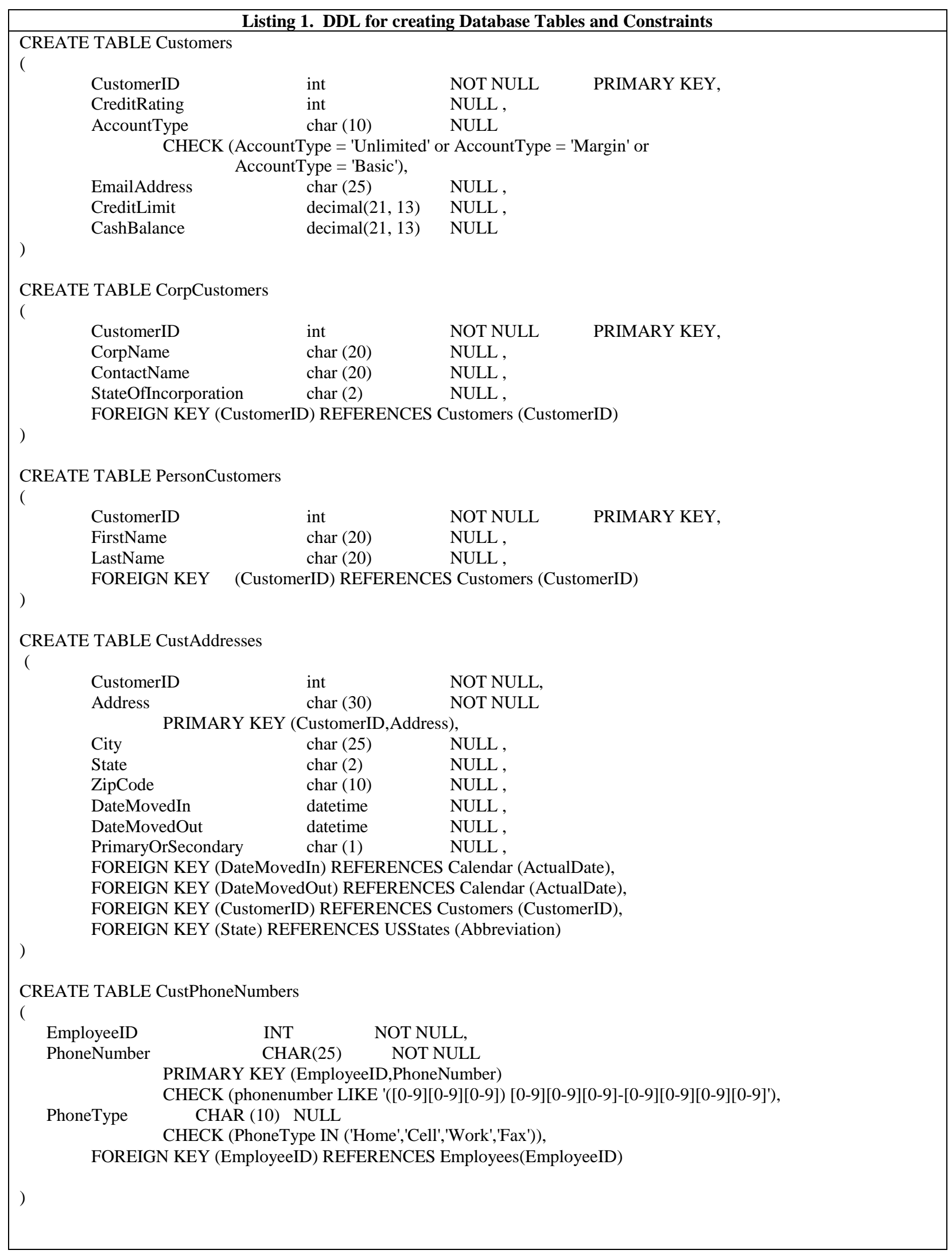




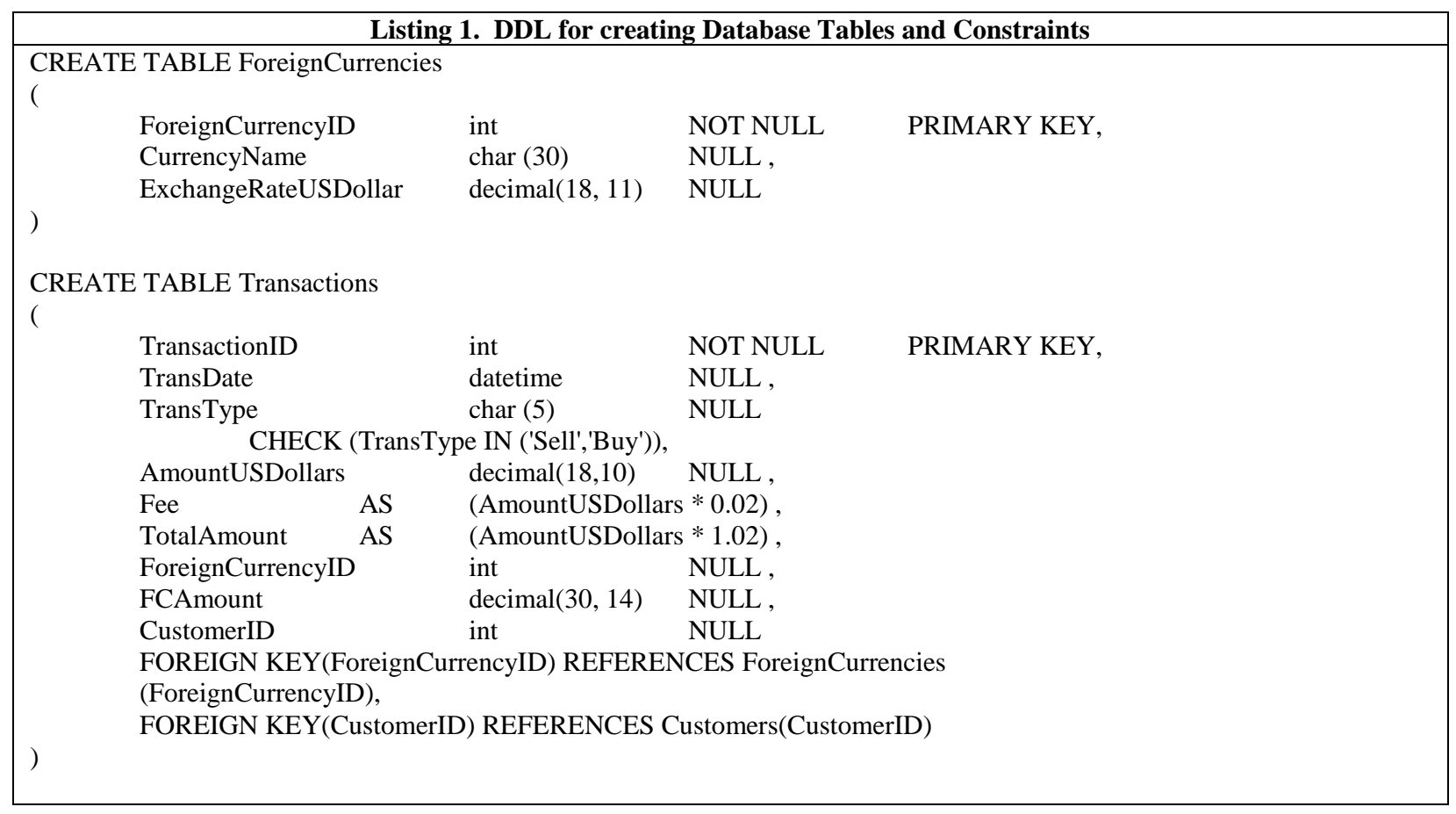

The Calendar table in the code listing above is a utility table used to identify weekdays/weekends, holidays, and days of the week. Although this information can be obtained using built-in SQL Server Date functions in queries, utilizing the Calendar table simplifies many of these operations, particularly for SQL beginners. The following listing provides the code for populating the calendar table with dates and associated information from January 1,1900 to December $31,2019^{3}$.

\section{Listing 2. SQL Code for populating the Calendar table}

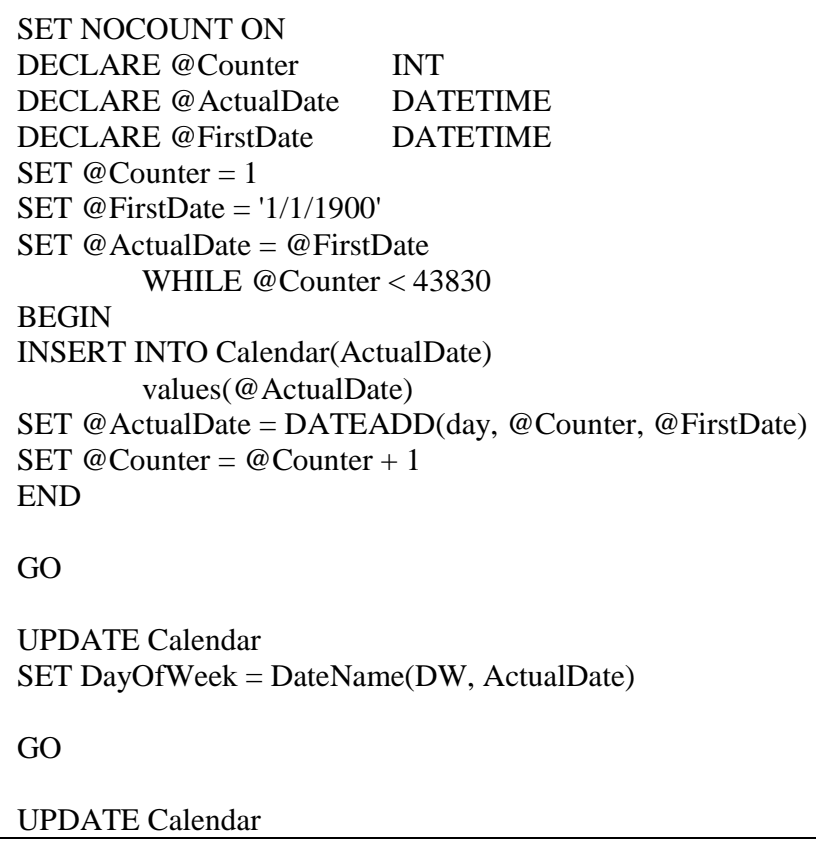

${ }^{3}$ Only three holidays are identified in our example. However, other holidays could be added if desired. 
Listing 2. SQL Code for populating the Calendar table

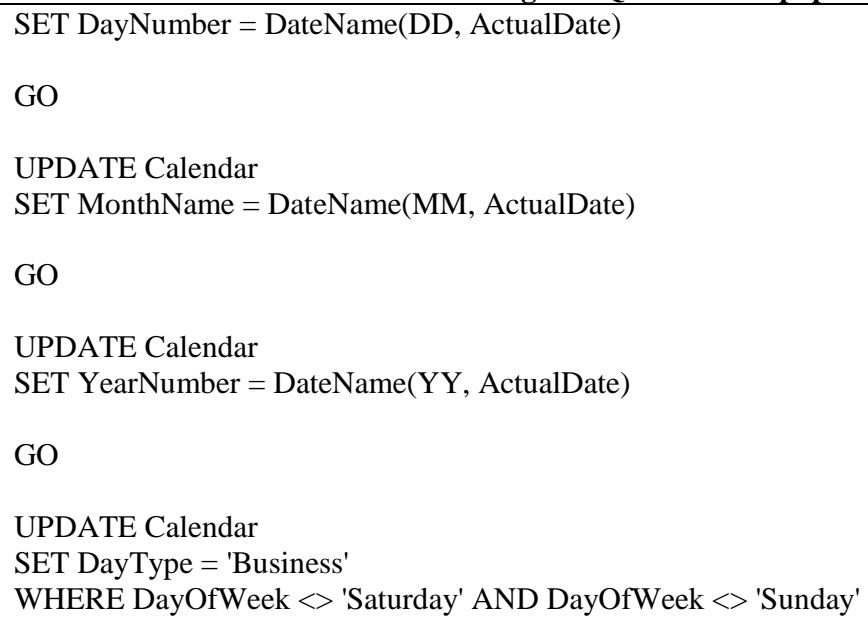

GO

When a new foreign currency transaction is inserted in the database or an existing transaction is modified, the foreign currency amount associated with the transaction can be calculated from the AmountUSDollars field of the Transactions table and the ExchangeRateUSDollar field of the ForeignCurrencies table. This is accomplished using a database trigger, the DDL for which is shown below in Listing 3. INSERTED is an SQL Server-proprietary pointer to the currently inserted tuple.

\section{Listing 3. DDL for the Foreign Currency Amount Trigger}

CREATE TRIGGER FCAmountCalc ON dbo.Transactions

FOR INSERT, UPDATE

AS

UPDATE Transactions

SET FCAmount $=$ t.AmountUSDollars $*$ ExchangeRateUSDollar

FROM INSERTED AS i JOIN ForeignCurrencies AS FC ON

(i.ForeignCurrencyID = FC.ForeignCurrencyID) JOIN Transactions AS t

ON $($ i. TransactionID $=$ t. TransactionID $)$ 


\section{WEB MODULES}

In this section, we outline the development of a Web application that interfaces with the foreign currency database. The application provides functionality supporting each of the CRUD operations for customers, addresses, phone numbers, and foreign currency transactions. The technology used in these examples is ASP.NET 3.5, with $\mathrm{C \#}$ as the programming language. All code was developed in Microsoft Visual Studio 2005 . We begin by discussing the advantages of a tiered application architecture and describing how such an architecture is applied to the foreign currency application. We then present code samples that illustrate the functionality of each tier.

\section{Tiered Application Architecture}

The growing complexity of today's software applications has prompted a movement toward software architectures that support component modularity and reusability. A well-known approach to promoting this objective is to develop a software application as a series of logical tiers, producing what is referred to as an $n$-tier architecture. Each tier encapsulates a logical set of functions within the application, and communicates with one or more other tiers through a well-defined interface. A tiered architecture provides enhanced flexibility and maintainability, since code within each tier can be modified independently of the other tiers within the application. A common version of the $n$-tier architecture employs three distinct application tiers: the data tier, the business tier, and the presentation tier. The data tier is responsible for managing connection to the data source and handling all data source interactions. The business tier interacts with the data tier and performs logical operations on the data retrieved. The business tier then passes the data on to the presentation tier, which is responsible for managing the user interface and displaying the appropriate data to the end user. Figure 2 provides an example of a user interacting with a tiered architecture in an e-commerce application.

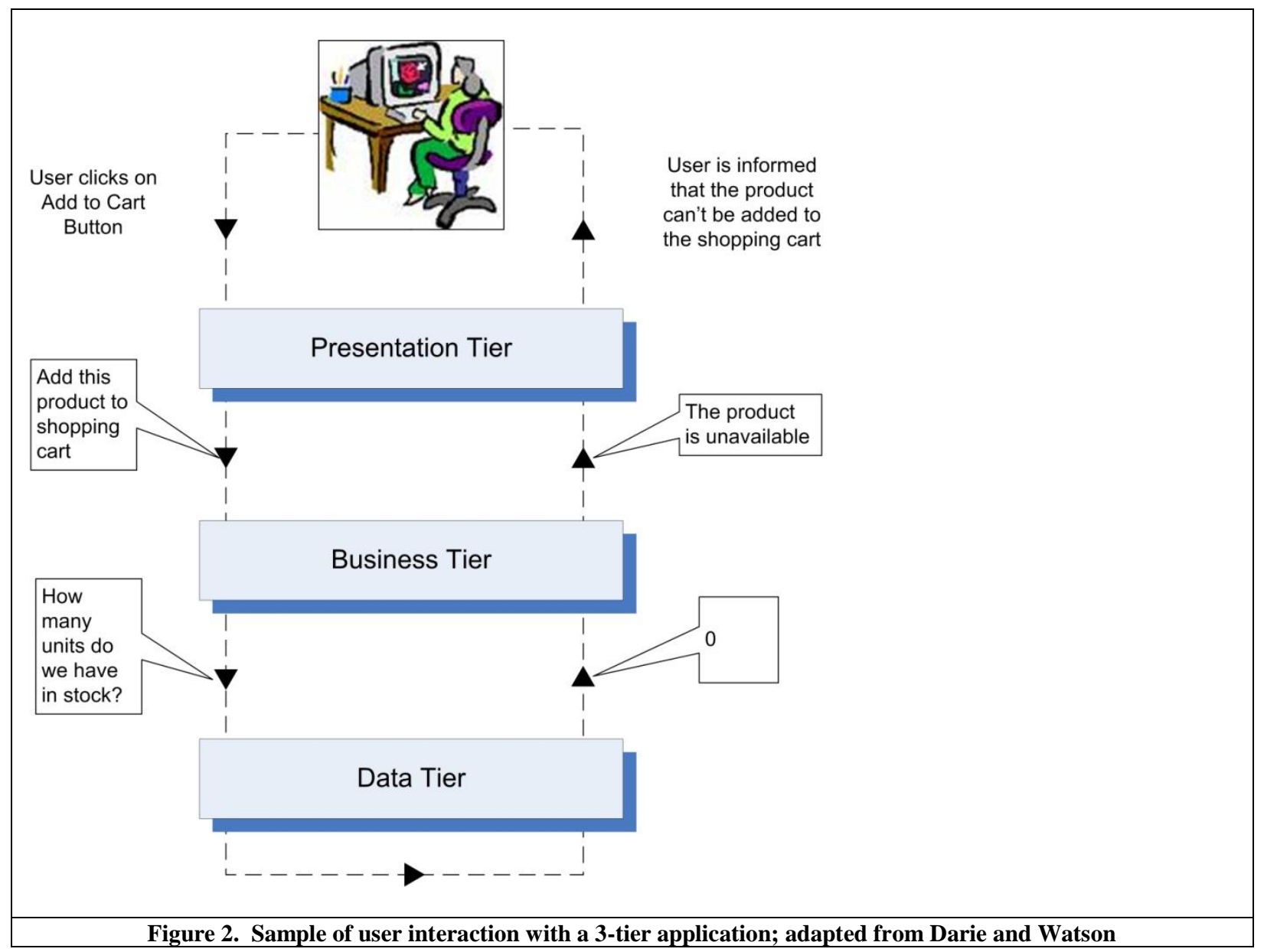


An overview of the 3-tier architecture used in the foreign currency application is shown in Figure 3. Each tier of this architecture is then described below.

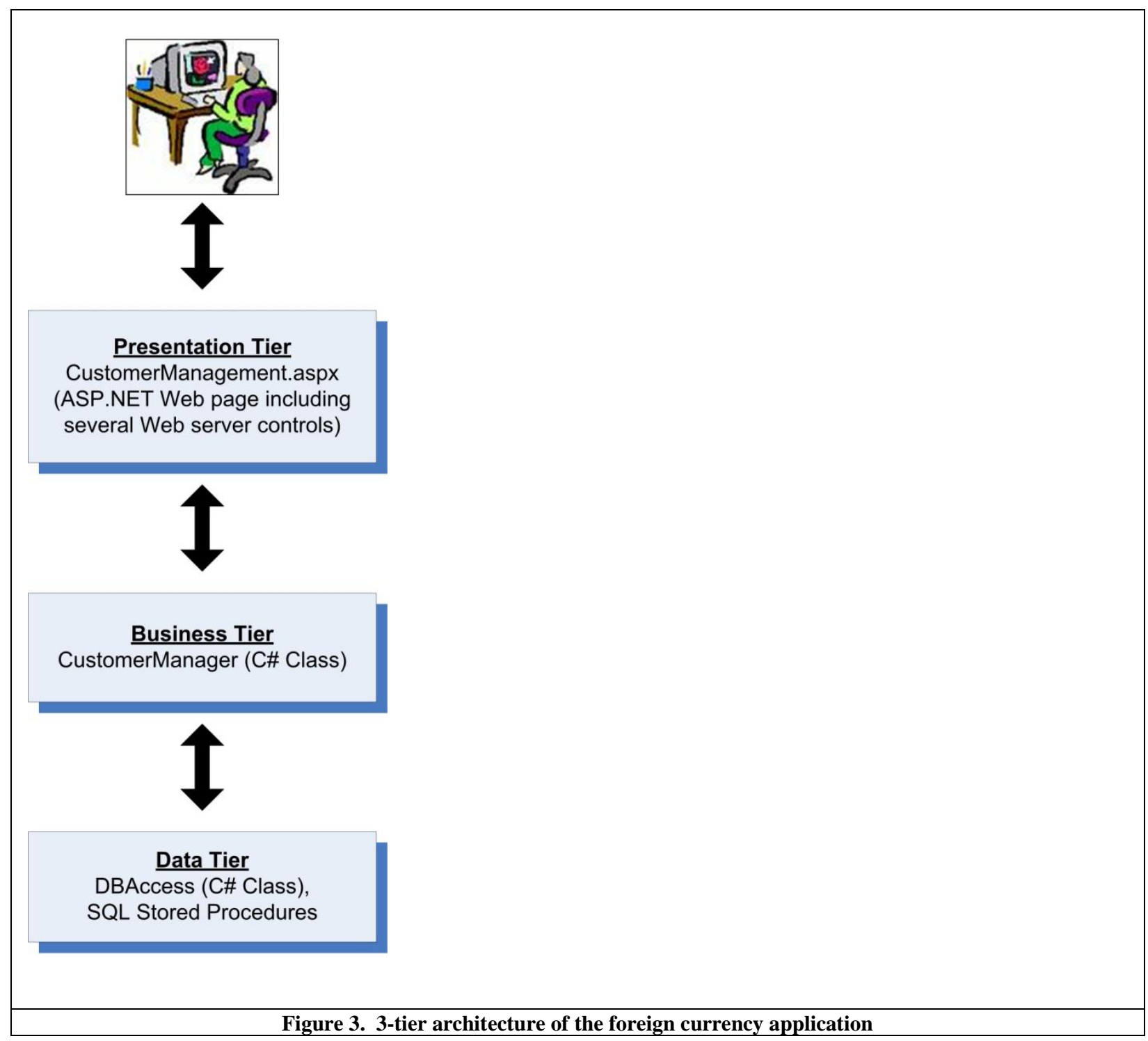

Data Tier

The purpose of the data tier is to manage interactions with a data source such as a relational database. A frequently recommended technique for handling these interactions is to encapsulate data manipulation operations within stored procedures, database objects that store code for performing one or many data operations. Because stored procedures are housed within and managed by the DBMS, they offer several performance and security benefits over sending SQL commands directly from application code [7]. Using stored procedures obviates the need to place potentially complex SQL statements directly within the application code, thus promoting code modularity and interpretability. For these reasons, stored procedures are well-suited to creating a tiered data-driven Web application. 
As shown in Figure 3, the data tier of the foreign currency application consists of SQL stored procedures and a single C\# class called DBAccess. The purpose of the DBAccess class is to: (1) receive database requests from the business tier, (2) connect to the database and invoke the required operations through stored procedure calls, and (3) return data to the business tier. The code for this class is shown in Listing 4. (Descriptive code comments within this and subsequent listings are enclosed in $/ * * /$.)

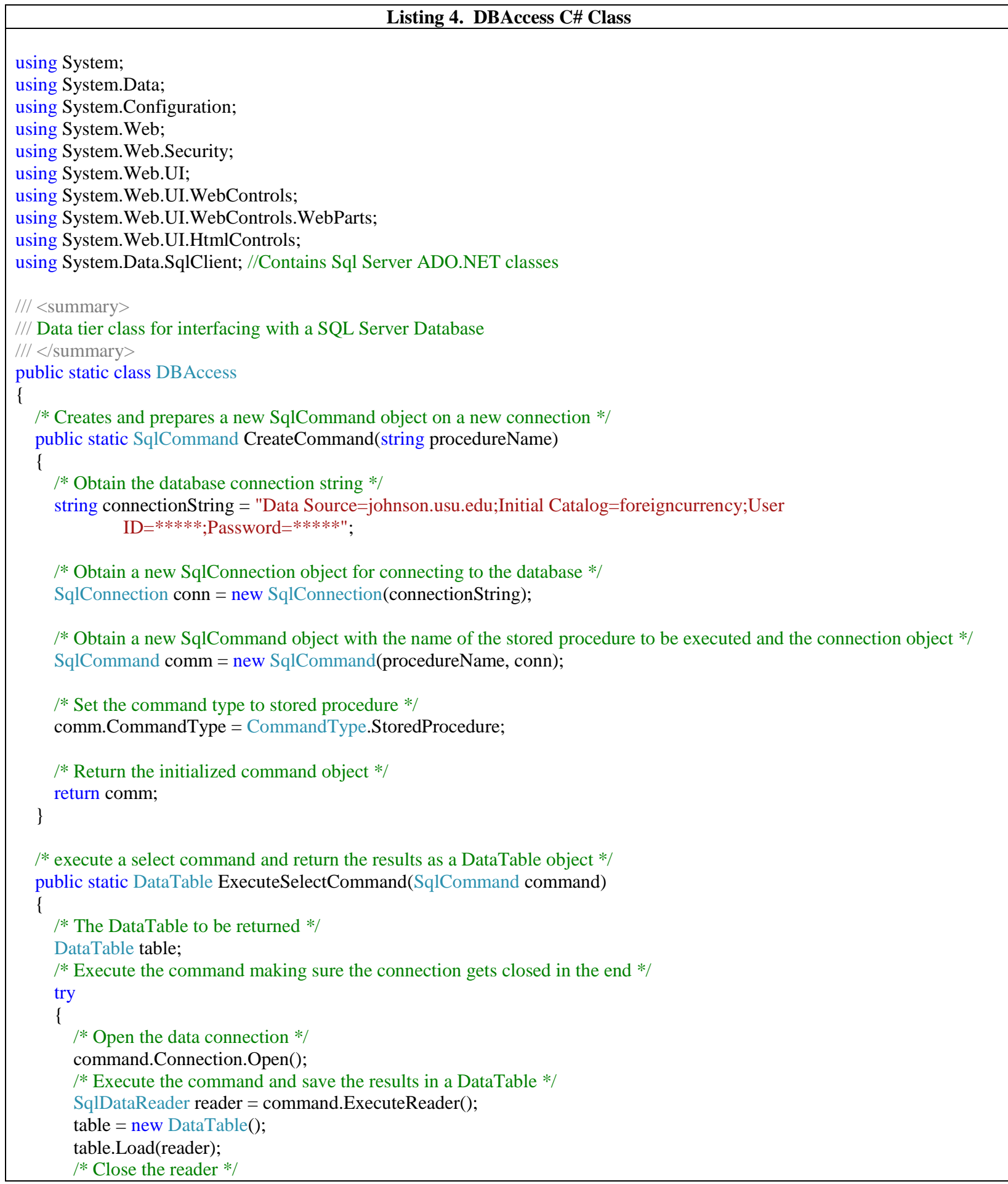




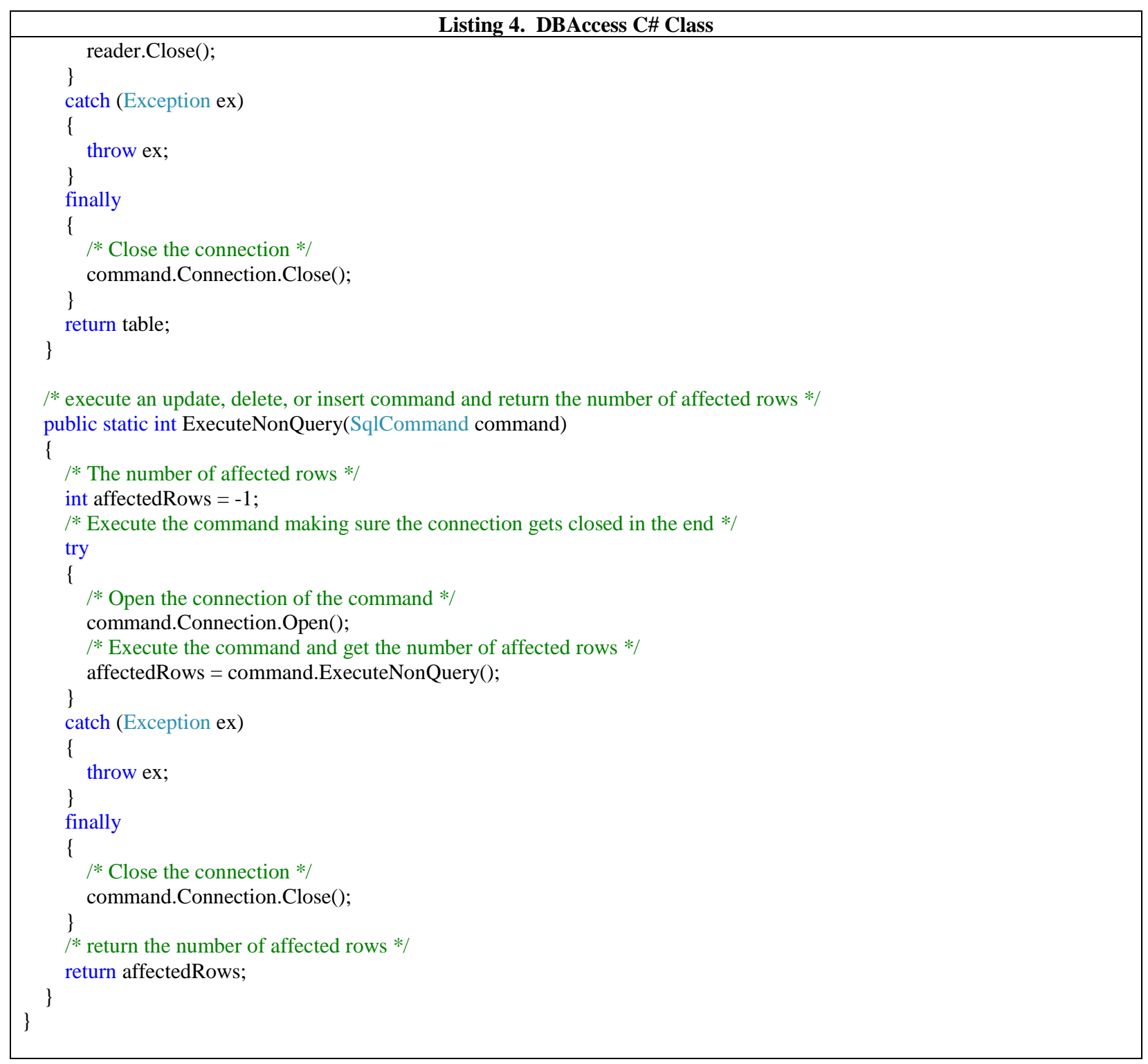

\section{Business Tier}

The function of the business tier is to serve as an interface with the data tier and the presentation tier and implement any required business logic in processing the data. The business tier of a moderately-sized application may consist of several application classes that manage these functions. For the foreign currency applicatoin, the business tier is implemented using a single static C\# class named CustomerManager. This class contains methods needed to select, insert, update, and delete all customer information, including transactions. In the interest of brevity, the entire CustomerManager class will not be presented here. Instead, sample methods from this class will be shown in the Application Examples section below.

\section{Presentation Tier}

As its name suggests, the presentation tier is responsible for receiving data from the business tier and presenting it to the application user. The presentation tier of a typical Web application consists of a collection of Web pages which are rendered to the user in a browser. For the foreign currency application, the presentation tier 
consists of a single ASP.NET Web page named CustomerManagement.aspx. Consistent with the recent ASP.NET code-behind development model ${ }^{4}$, this page is logically divided into two files. The first, CustomerManagement.aspx, contains all declarative HTML and ASP.NET markup that is rendered to the browser as HTML. This file is referred to as the content file. The second, CustomerManagement.aspx.cs, contains all of the programming logic required to respond to various events associated with the application. This file is called the code-behind file, since it sits "behind" the content file and responds to events that occur on the page. When the user requests an .aspx page from the Web server, the content and code-behind files are combined to create a fully functional Web page that is rendered to the user.

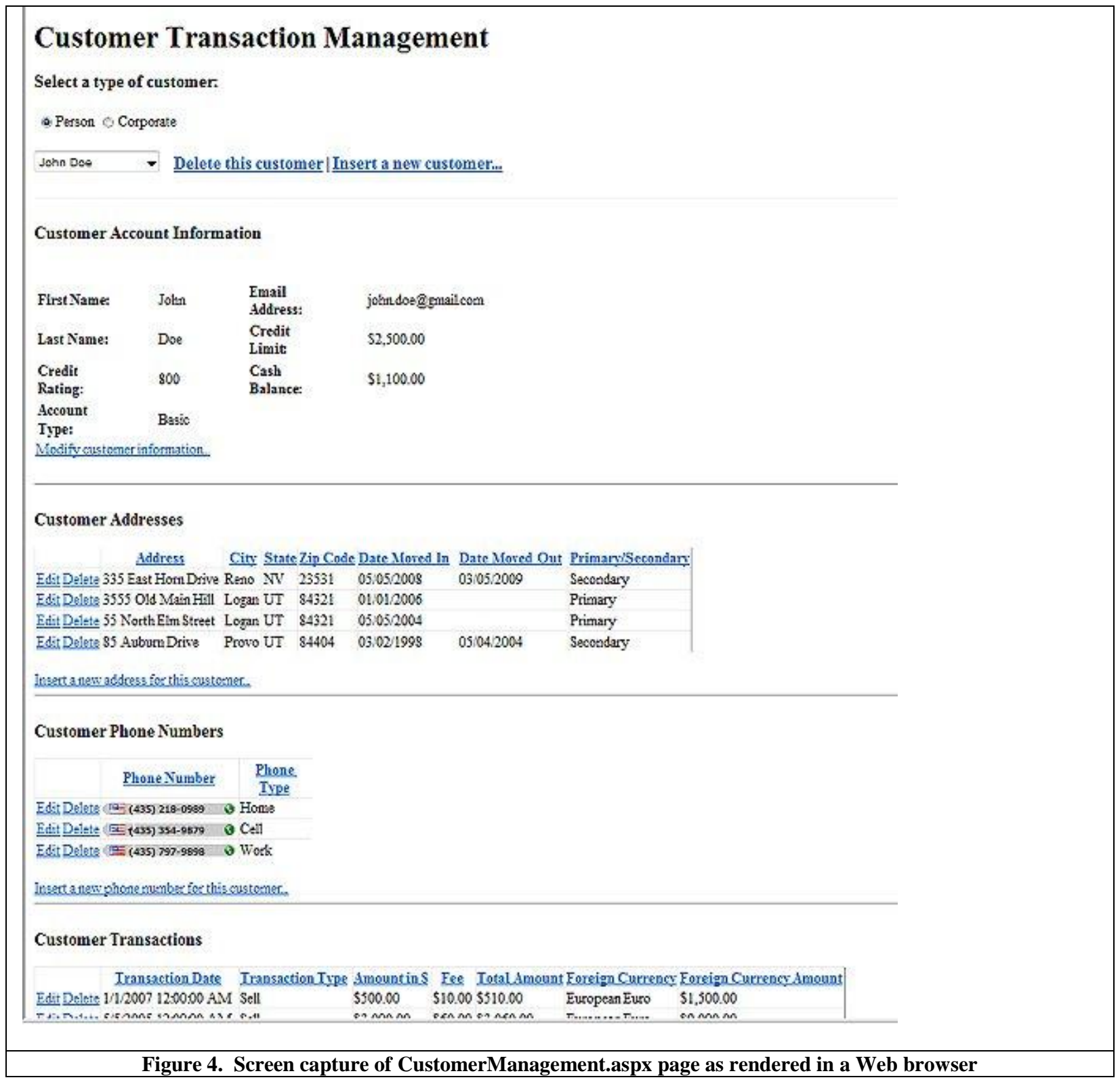

\footnotetext{
${ }^{4}$ For a more detailed discussion of the code-behind model, see http://quickstarts.asp.net/QuickStartv20/aspnet/doc/pages/codebehind.aspx.
} 
A screen capture of the CustomerManager.aspx page is shown in Figure $4^{5}$. As with the business tier class, the code for this page will not be presented here in its entirety; however, relevant components of the page will be shown in the next section, where we present sample code for performing CRUD operations in the foreign currency application.

\section{Application Examples}

As noted above, the foreign currency application enables select, insert, update, and delete operations for customer information, including customer account details, addresses, phone numbers, and transactions. Table 1 summarizes each of the operations supported and identifies the relevant components of each tier. In the interest of parsimony, only components in shaded rows of Table 1 are described further below. These components include:

1. Selecting person/corporate customer names and IDs from the database, and

2. Performing each of the CRUD operations for customer foreign currency transactions. application ${ }^{6}$.

These operations were chosen because they are illustrative of the other data operations supported by the

\footnotetext{
${ }^{5}$ We intentionally minimized formatting of the CustomerManagement.aspx page so as not to distract from database connectivity as the primary focus of this module. However, the design of the page could be altered using ASP.NET themes and skins and/or Cascading Style Sheets (CSS).

${ }^{6}$ Code for the entire foreign currency application is available online at: http://olsen.usu.edu/RBIS/RBISCRUDontheWeb.html
} 
Table 1. Data operations and application components of the foreign currency application

\begin{tabular}{|c|c|c|c|c|}
\hline $\begin{array}{l}\text { DB } \\
\text { Operation }\end{array}$ & Data & $\begin{array}{l}\text { Data Tier } \\
\text { (Includes DBAccess class and the } \\
\text { following stored procedures) }\end{array}$ & $\begin{array}{l}\text { Business Tier } \\
\text { (Methods of the CustomerManager } \\
\text { class) }\end{array}$ & $\begin{array}{l}\text { Presentation Tier } \\
\text { (Controls of the CustomerManagement.aspx } \\
\text { page) }\end{array}$ \\
\hline \multirow[t]{6}{*}{ Select } & $\begin{array}{l}\text { All customer names and IDs for } \\
\text { person/corporate customers }\end{array}$ & GetPersonCustomers, GetCorpCustomers & $\begin{array}{l}\text { GetPersonCustomers, } \\
\text { GetCorpCustomers }\end{array}$ & DropDownList ddlCustomers \\
\hline & Customer account information & $\begin{array}{l}\text { GetPersonCustomerInfo, } \\
\text { GetCorpCustomerInfo }\end{array}$ & $\begin{array}{l}\text { GetPersonCustomerInfo, } \\
\text { GetCorpCustomerInfo }\end{array}$ & $\begin{array}{l}\text { FormView fvwPersonCustInfo, FormView } \\
\text { fvwCorpCustInfo, } \\
\text { ObjectDataSource odsCustInfo }\end{array}$ \\
\hline & Customer addresses & GetCustomerAddresses & GetCustomerAddresses & $\begin{array}{l}\text { GridView gvwCustAddresses, } \\
\text { ObjectDataSource odsCustAddresses }\end{array}$ \\
\hline & Customer phone numbers & GetCustomerPhoneNumbers & GetCustomerPhoneNumbers & $\begin{array}{l}\text { GridView gvwCustPhoneNumbers, } \\
\text { ObjectDataSource odsCustPhoneNumbers }\end{array}$ \\
\hline & Customer transactions & GetCustomerTransactions & GetCustomerTransactions & $\begin{array}{l}\text { GridView gvwCustTransactions, } \\
\text { ObjectDataSource odsCustTransactions }\end{array}$ \\
\hline & Foreign currency names and IDs & GetForeignCurrencies & GetForeignCurrencies & $\begin{array}{l}\text { DropDownList ddlEditForeignCurrency, } \\
\text { DropDownList ddlInsertForeignCurency }\end{array}$ \\
\hline \multirow[t]{4}{*}{ Insert } & $\begin{array}{l}\text { Person/corporate customer, } \\
\text { including account information }\end{array}$ & $\begin{array}{l}\text { InsertCustomer, InsertPersonCustomer, } \\
\text { InsertCorpCustomer }\end{array}$ & $\begin{array}{l}\text { InsertPersonCustomer, } \\
\text { InsertCorpCustomer }\end{array}$ & $\begin{array}{l}\text { FormView fvwPersonCustInfo, FormView } \\
\text { fvwCorpCustInfo, } \\
\text { ObjectDataSource odsCustInfo }\end{array}$ \\
\hline & Customer address & InsertCustomerAddress & InsertCustomerAddress & $\begin{array}{l}\text { DetailsView dvwCustAddresses, } \\
\text { ObjectDataSource odsCustAddresses }\end{array}$ \\
\hline & Customer phone number & InsertCustomerPhoneNumber & InsertCustomerPhoneNumber & $\begin{array}{l}\text { DetailsView dvwCustPhoneNumbers, } \\
\text { ObjectDataSource odsCustPhoneNumbers }\end{array}$ \\
\hline & Customer transaction & InsertCustomerTransaction & InsertCustomerTransaction & $\begin{array}{l}\text { DetailsView dvwCustTransactions, } \\
\text { ObjectDataSource odsCustTransactions }\end{array}$ \\
\hline \multirow[t]{4}{*}{ Update } & Customer account information & $\begin{array}{l}\text { UpdateCustomerInfo, } \\
\text { UpdatePersonCustomerInfo, } \\
\text { UpdateCorpCustomerInfo }\end{array}$ & $\begin{array}{l}\text { UpdatePersonCustomerInfo, } \\
\text { UpdateCorpCustomerInfo }\end{array}$ & $\begin{array}{l}\text { FormView fvwPersonCustInfo, FormView } \\
\text { fvwCorpCustInfo, } \\
\text { ObjectDataSource odsCustInfo }\end{array}$ \\
\hline & Customer address & UpdateCustomerAddress & UpdateCustomerAddress & $\begin{array}{l}\text { GridView gvwCustAddresses, } \\
\text { ObjectDataSource odsCustAddresses }\end{array}$ \\
\hline & Customer phone number & UpdateCustomerPhoneNumber & UpdateCustomerPhoneNumber & $\begin{array}{l}\text { GridView gvwCustPhoneNumbers, } \\
\text { ObjectDataSource odsCustPhoneNumbers }\end{array}$ \\
\hline & Customer transaction & UpdateCustomerTransaction & UpdateCustomerTransaction & $\begin{array}{l}\text { GridView gvwCustTransactions, } \\
\text { ObjectDataSource odsCustTransactions }\end{array}$ \\
\hline \multirow[t]{4}{*}{ Delete } & $\begin{array}{l}\text { Person/corporate customer, } \\
\text { including account information }\end{array}$ & $\begin{array}{l}\text { DeletePersonCustomer, } \\
\text { DeleteCorpCustomer }\end{array}$ & $\begin{array}{l}\text { DeletePersonCustomer, } \\
\text { DeleteCorpCustomer }\end{array}$ & LinkButton btnDeleteCustomer \\
\hline & Customer address & DeleteCustomerAddress & DeleteCustomerAddress & $\begin{array}{l}\text { GridView gvwCustAddresses, } \\
\text { ObjectDataSource odsCustAddresses }\end{array}$ \\
\hline & Customer phone number & DeleteCustomerPhoneNumbers & DeleteCustomerPhoneNumbers & $\begin{array}{l}\text { GridView gvwCustPhoneNumbers, } \\
\text { ObjectDataSource odsCustPhoneNumbers }\end{array}$ \\
\hline & Customer transaction & DeleteCustomerTransaction & DeleteCustomerTransaction & $\begin{array}{l}\text { GridView gvwCustTransactions, } \\
\text { ObjectDataSource odsCustTransactions }\end{array}$ \\
\hline
\end{tabular}




\section{Selecting Customer IDs and Names for Person/Corporate Customers}

In order to view or modify customer information, the application user must first select a customer type (person or corporate), and then a specific customer (see Figure 4). This is accomplished with two ASP.NET server controls: a RadioButtonList named rblCustType and a DropDownList named ddlCustomer. The user first selects a customer type (person or corporate) from the rblCustType control. When a selection is made, the ddlCustomer control is populated with a list of person or corporate customers according to the selected type in rblCustType. The ddlCustomer control shows a list of customer names (for person customers) or corporation names (for corporate customers). The Value property of each item in the DropDownList contains the CustomerID of the customer.

The data tier for supporting this functionality consists of two stored procedures in addition to the DBAccess class presented earlier. These stored procedures, shown in Listing 5, provide parallel functionality for person and corporate customers, respectively.

\begin{tabular}{|l|}
\hline Listing 5. Data Tier - DDL for GetPersonCustomers and GetCorpCustomers Stored Procedures \\
\hline CREATE PROCEDURE GetPersonCustomers \\
AS \\
SELECT CustomerID, FirstName + ' ' + LastName AS FullName \\
FROM PersonCustomers \\
ORDER BY LastName; \\
CREATE PROCEDURE GetCorpCustomers \\
AS SELECT CustomerID, CorpName \\
FROM CorpCustomers \\
ORDER BY CorpName;
\end{tabular}

The business tier components for supporting this operation include two methods of the CustomerManager class: GetPersonCustomers and GetCorpCustomers. These methods, shown in Listing 6, utilize the DBAccess data tier class to call the stored procedures listed above. They then pass the resulting DataTable object back to the presentation tier.

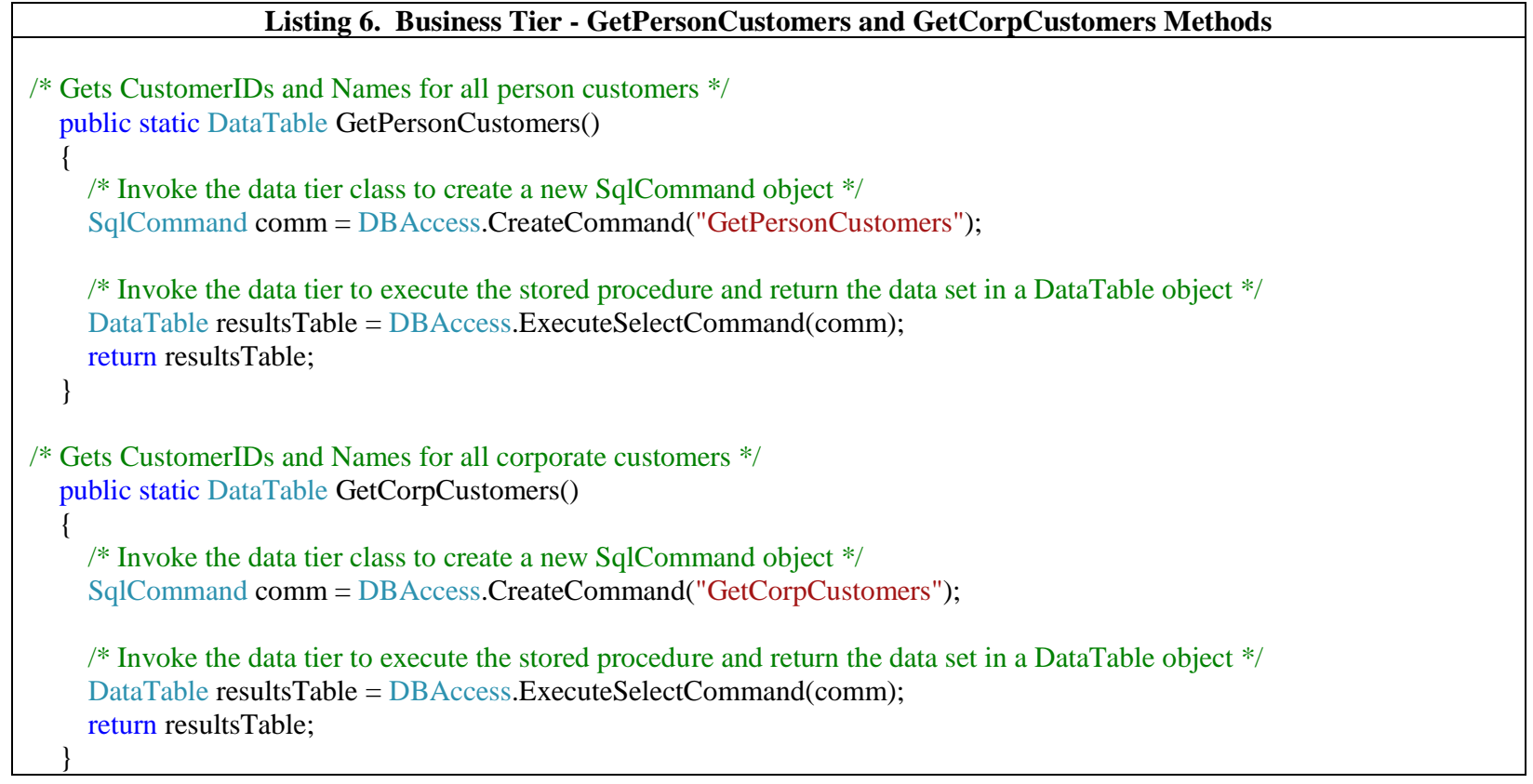


As described above, the presentation tier involves two ASP.NET server controls: a RadioButtonList named rblCustType and a DropDownList named ddlCustomer. The content file code for these controls is shown in Listing 7. Note that the DataValueField property of ddlCustomer is set to CustomerID. This causes the value of each item in the DropDownList control to be set to the value stored in the CustomerID column of the control's data source.

\begin{tabular}{l} 
Listing 7. Presentation Tier - ddlCustomers DropDownList Content File Code \\
\hline \\
<asp:RadioButtonList ID="rblCustType" runat="server" AutoPostBack="true" \\
OnSelectedIndexChanged="rblCustType_SelectedIndexChanged" \\
RepeatDirection="Horizontal"> \\
<asp:ListItem Value="0">Person</asp:ListItem> \\
<asp:ListItem Value="1">Corporate</asp:ListItem> \\
</asp:RadioButtonList> \\
<asp:DropDownList ID="ddlCustomer" runat="server" DataValueField="CustomerID" \\
$\begin{array}{l}\text { OnSelectedIndexChanged="ddlCustomer_SelectedIndexChanged" AutoPostBack="True" AppendDataBoundItems="True"> } \\
\text { </asp:DropDownList> }\end{array}$
\end{tabular}

The data source of ddlCustomer is not set declaratively in the content file. Instead, the data source is set dynamically in the code-behind file depending on whether the application user has selected person or corporate customers in rblCustType. As shown in Listing 8, a private method, initializeCustomerddl, is called to initialize the DropDownList whenever the selected customer type in rblCustType is changed. Note that this method sets the data source of ddlCustomer by calling the appropriate business tier method, depending on the type of customer selected. The visibility of several panels is also set in this method; this is simply to ensure that only certain components of the page are made visible before the user has selected a specific customer.

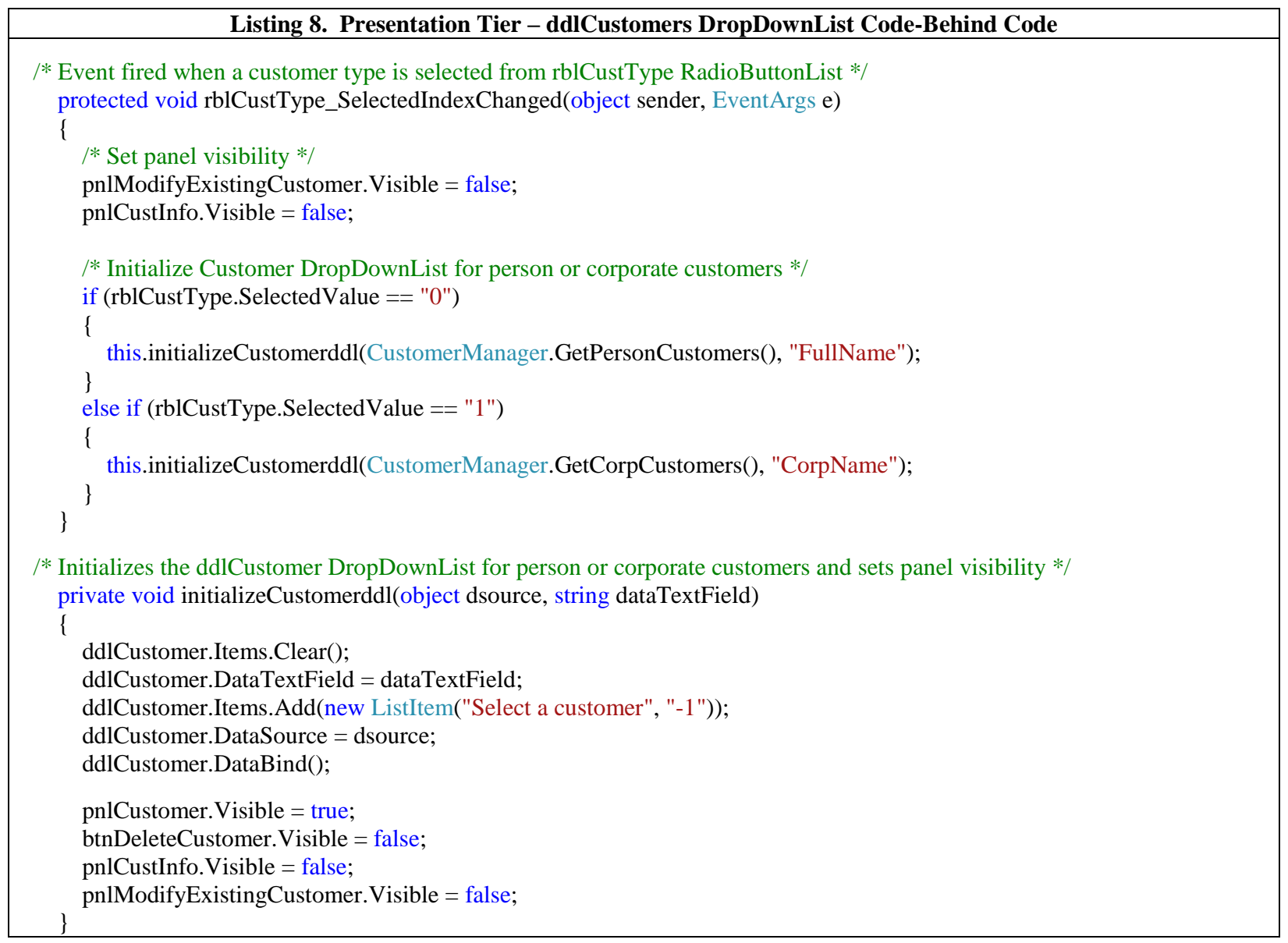


Selecting, Inserting, Updating, and Deleting Customer Transaction Data

After selecting a customer type from rblCustType and a specific customer from ddlCustomer, the application user can then view, insert, update, and delete customer account information, addresses, phone numbers, and transactions. We present here the code for viewing and manipulating customer transactions, as it is exemplary of the code for viewing and manipulating the other types of customer data.

The data tier supporting customer transactions consists of four stored procedures for performing each of the CRUD operations. DDL for these stored procedures is shown in Listing 9. We point out that the InsertCustomerTransaction procedure creates a new primary key value for the record to be inserted by selecting the maximum key value of the table and incrementing it by one. Although this can be accomplished using an IDENTITY field in SQL Server, IDENTITY implementations differ across DBMSs and can cause portability issues when the database is migrated[1]. We therefore chose the approach below to maximize portability across DBMSs.

In addition to the four stored procedures described above, an additional stored procedure is utilized to select all foreign currency names and IDs from the ForeignCurrencies lookup table. The data returned by this procedure will be utilized to support updating and inserting transactions, as will be shown below.

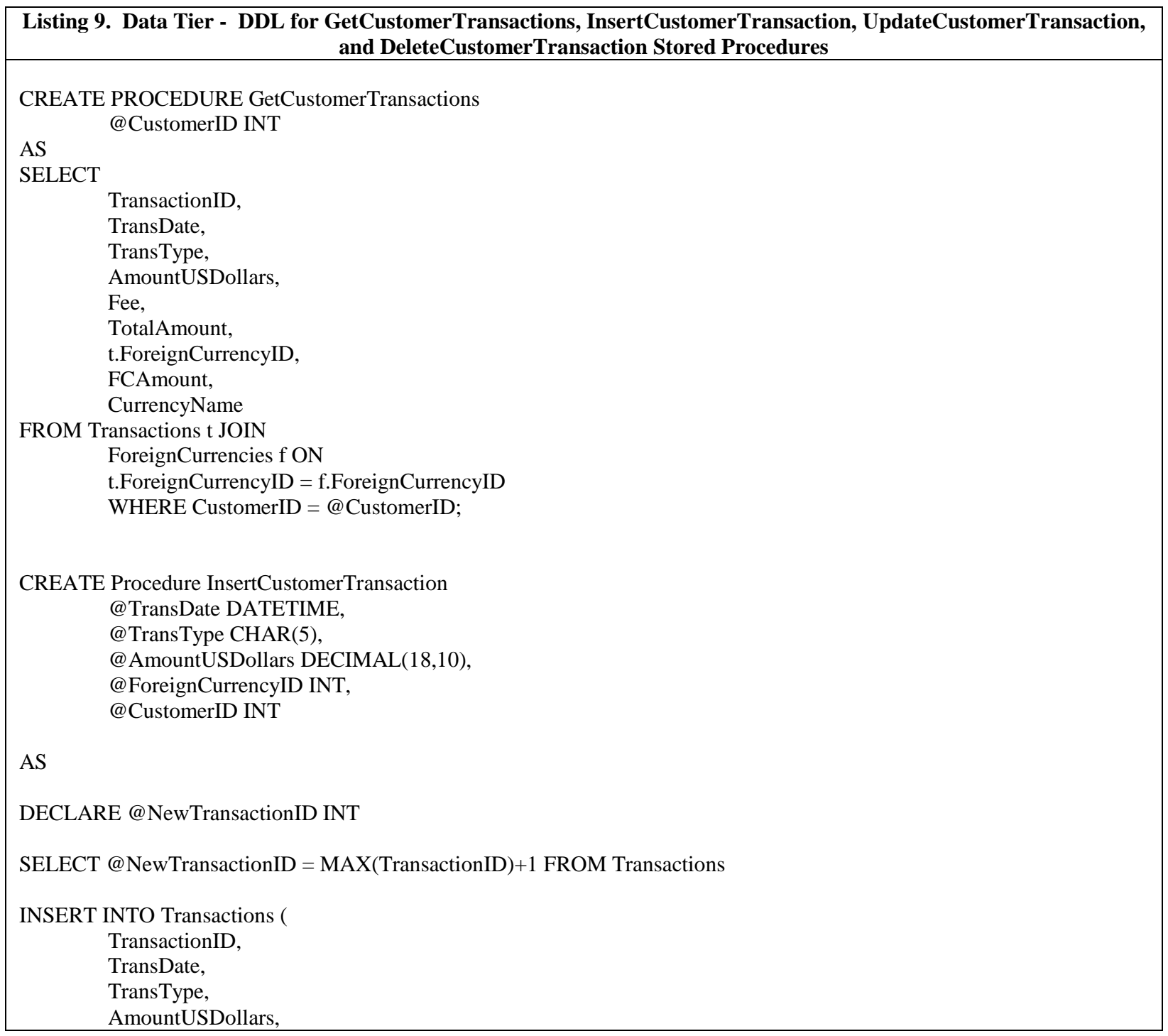




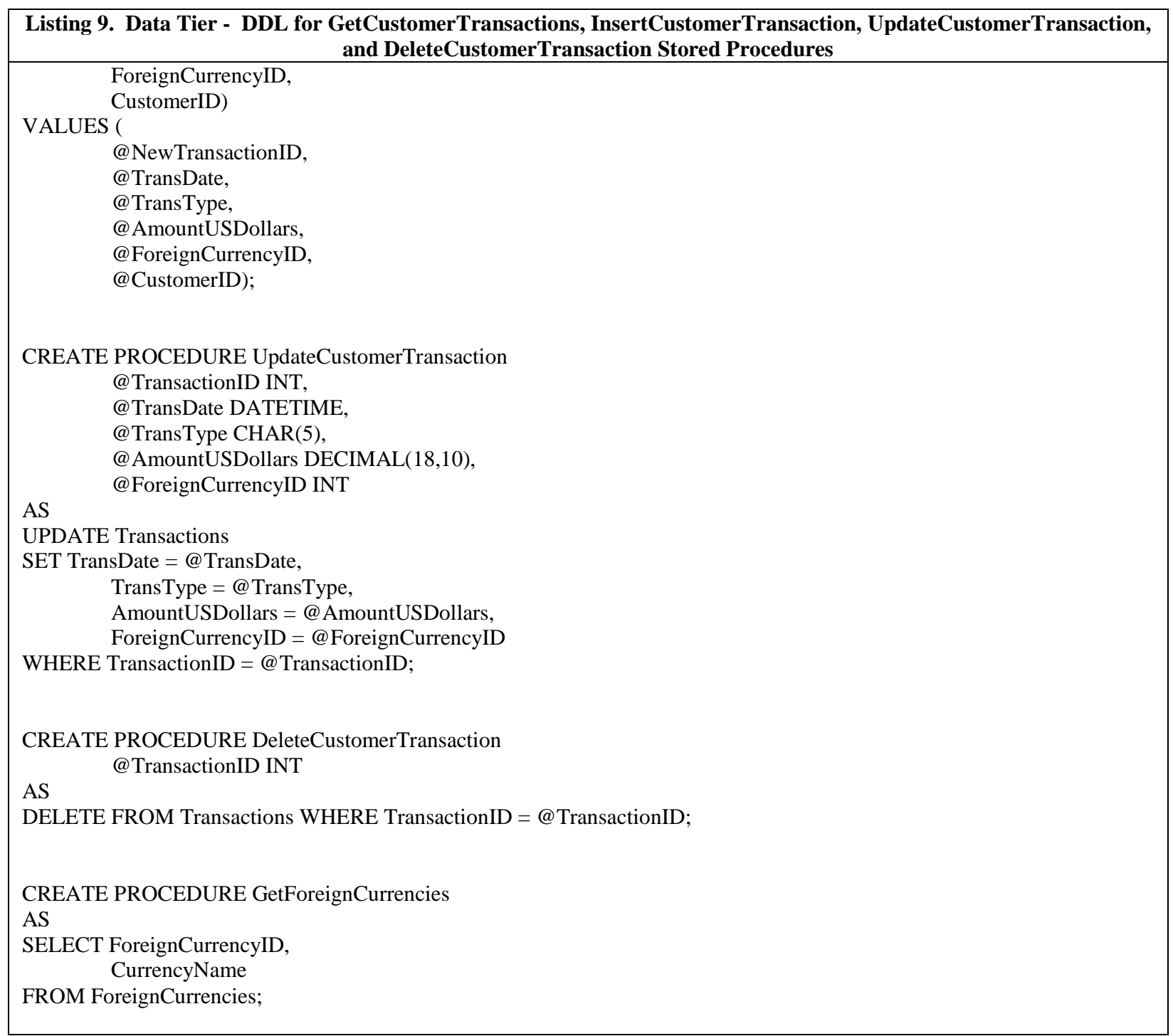

As in the previous example, the business tier supporting transaction management consists of methods of the CustomerManager class that invoke the data tier and pass data on to the presentation tier. These methods, one corresponding to each of the stored procedures above, are shown in Listing 10.

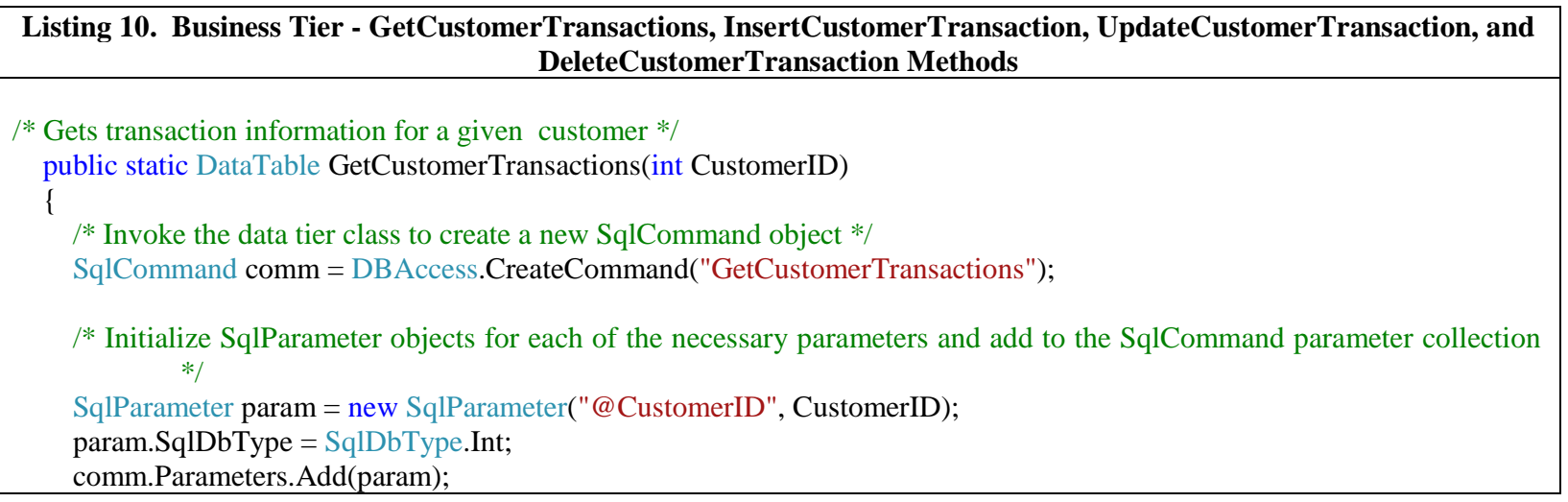




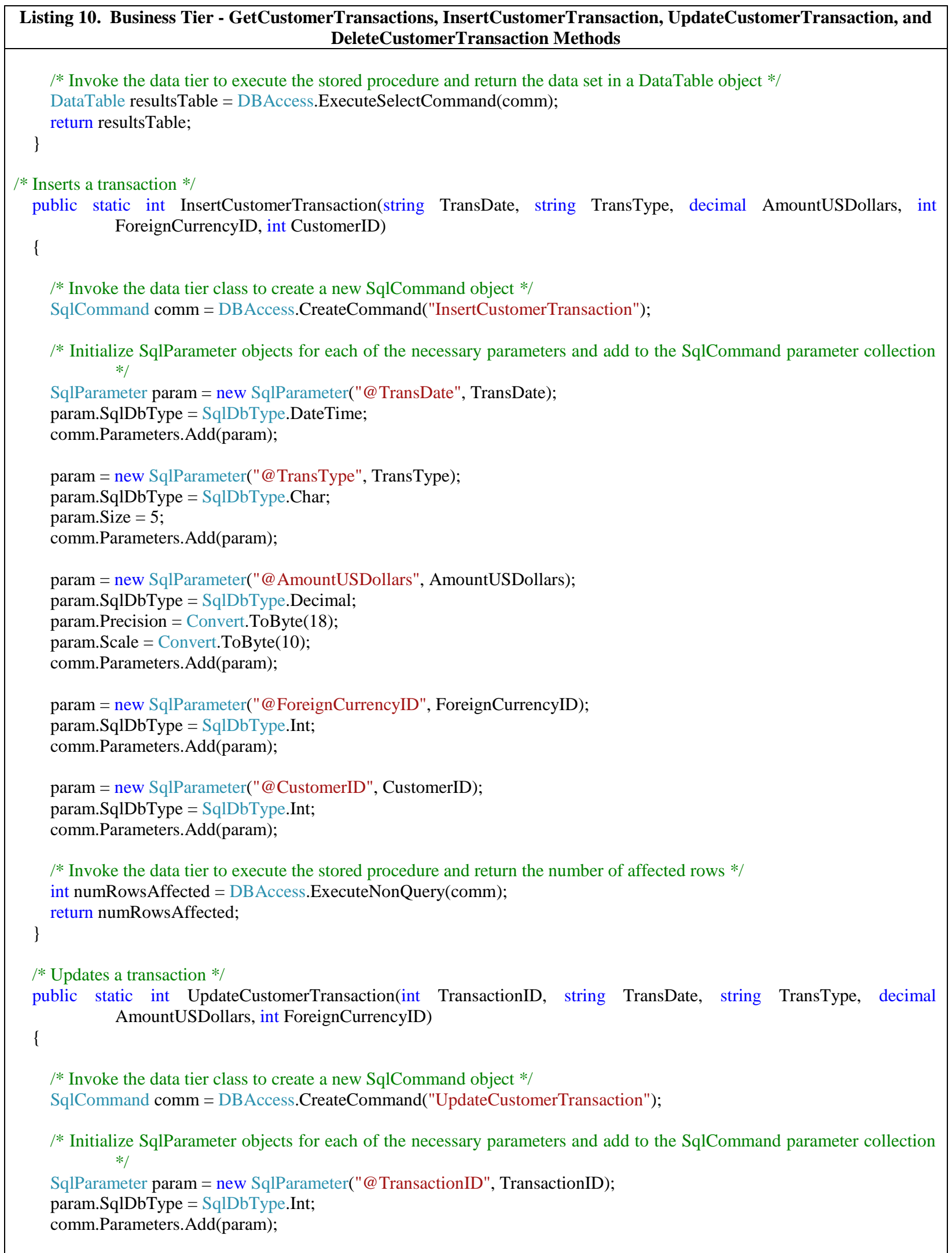




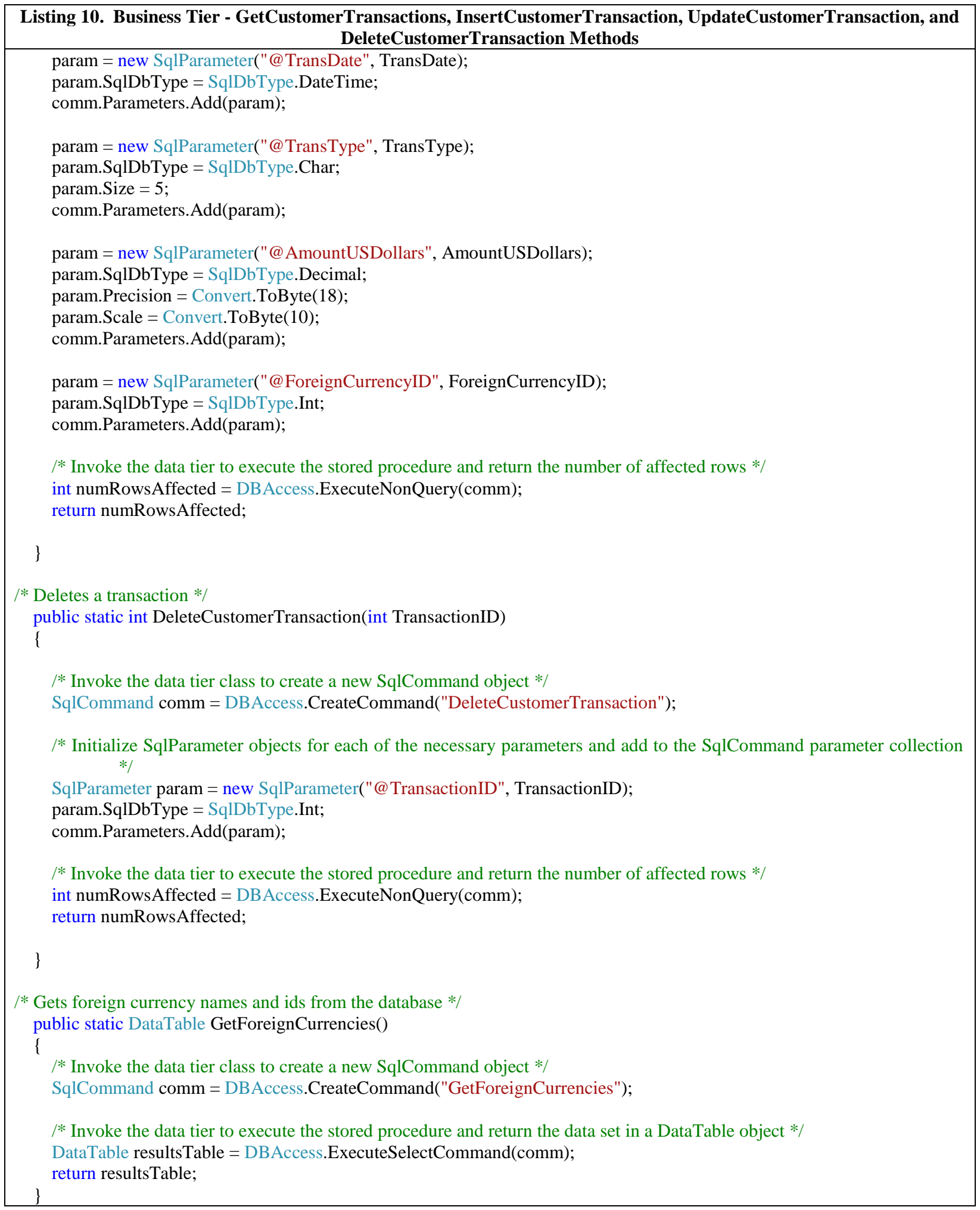

The architecture of the presentation tier differs somewhat from that of the previous example in that the present case utilizes an ObjectDataSource control to handle interactions with the business tier. The 
ObjectDataSource control provides means for interfacing with a business object such as the CustomerManager object in the present example. The ObjectDataSource is connected to a business tier object by setting its TypeName and Method properties, the former indicating the business tier class to be used and the latter indicating a method for performing each of the four CRUD operations. Additionally, parameters required by each of these methods can be specified.

The content file code for the odsCustTransactions ObjectDataSource is provided in Listing 11. The TypeName property indicates the CustomerManager business object, and the SelectMethod, InsertMethod, UpdateMethod, and DeleteMethod properties are set to the appropriate respective methods of this object. Parameters required by each of these methods are also specified. Note that the customerID parameter is implemented using a ControlParameter object that references the ddlCustomer DropDownList shown earlier. This specifies that the value of this parameter is retrieved from the selected value (i.e. the selected CustomerID) of the ddlCustomer DropDownList.

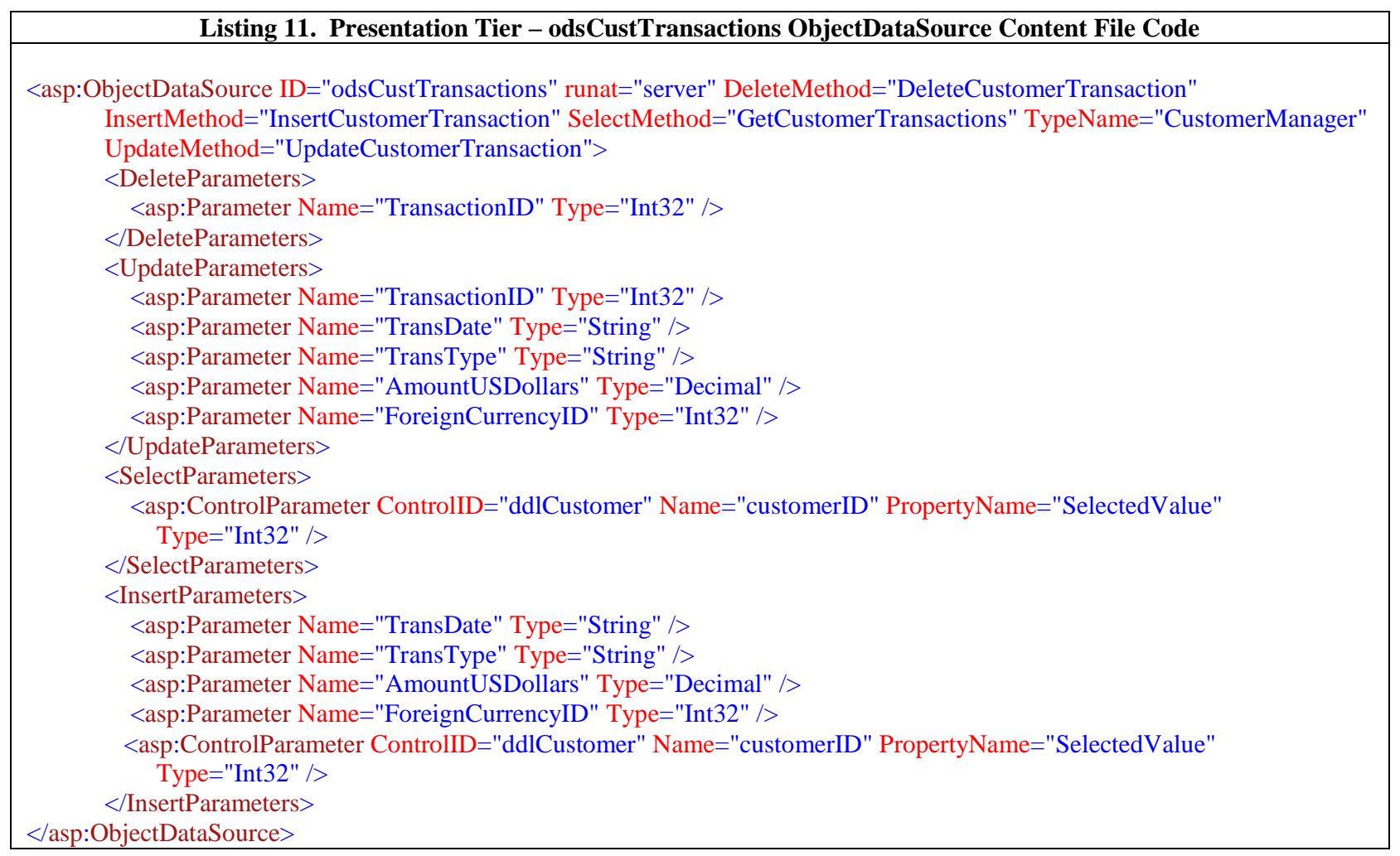

The ObjectDataSource provides an interface for interacting with the business tier, but it does not display the data to the application's user. For this, two ASP.NET data display controls are used. The first is a GridView control named gvwCustTransactions. The GridView control is useful for displaying data in tabular format, where the columns represent fields and the rows represent records in the data source. The GridView can be linked directly to a data source control such as the ObjectDataSource, and can provide "out of the box" select, update, and delete capabilities, significantly streamlining the code required to implement this functionality.

The content file code for gvwCustTransactions is shown in Listing 12. The GridView is a fairly complex control, and a full explanation of its intricacies will not be provided here. However some attributes shown in the code below deserve mention. First, the DataSourceID property indicates that the GridView will utilize the odsCustTransactions ObjectDataSource control as its data source. The DataKeyNames property points to the primary key of the data set returned by this data source. Second, the content (or columns) of the GridView is made up of a series of fields, including CommandFields, BoundFields, and TemplateFields. The CommandField provides edit and delete functionality, while the BoundFields and TemplateFields are linked to fields in the data source, as 
denoted by the DataField property (for BoundFields), and the Bind() method (for TemplateFields). Finally, note that the DataSource property of the ddlEditForeignCurrency DropDownList (contained within the foreign currency TemplateField) is set with a direct call to the business tier method GetForeignCurrencies, which returns a DataTable object to which the DropDownList can be bound.

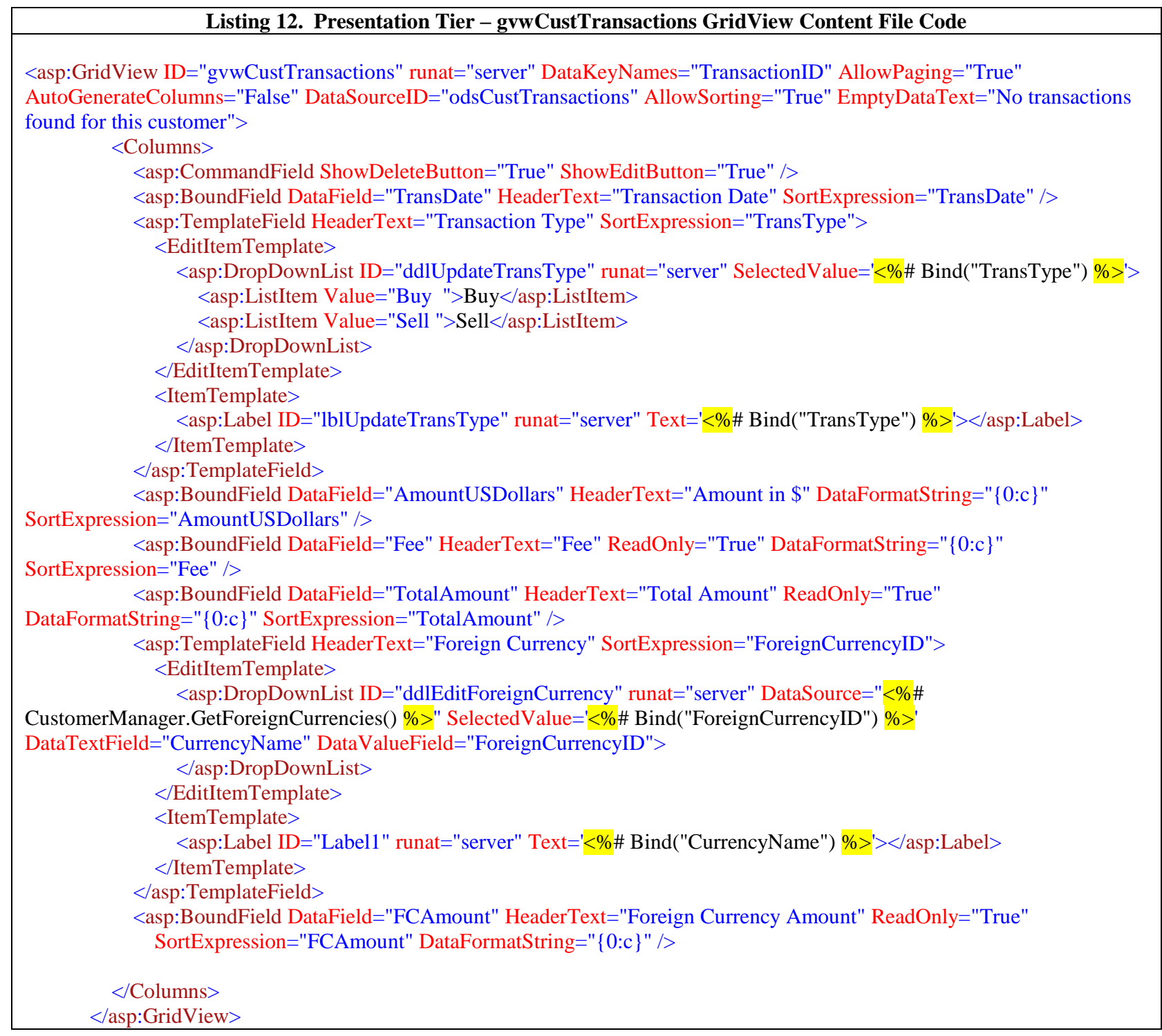

While the GridView control is extremely useful for selecting, updating, and deleting data records, it is not well suited for inserting new records. For this, a DetailsView control is used. A DetailsView is similar to a GridView in that it can be linked to an ObjectDataSource control and it displays data in a structured format. However, unlike the GridView, the DetailsView displays only one data record at a time and provides "out of the box" functionality for inserting a new record.

The content file code for the DetailsView dvwCustTransactions is shown in Listing 13. The code for this DetailsView is structurally similar to that of the gvwCustTransactions GridView described above, the primary difference being that the DetailsView contains a collection of fields rather than columns. Additionally, note that the DefaultMode property of the DetailsView is set to "Insert". This property is so set because the DetailsView is used 
only as a mechanism for inserting a new transaction, while the GridView is used to select, update, and delete existing transactions.

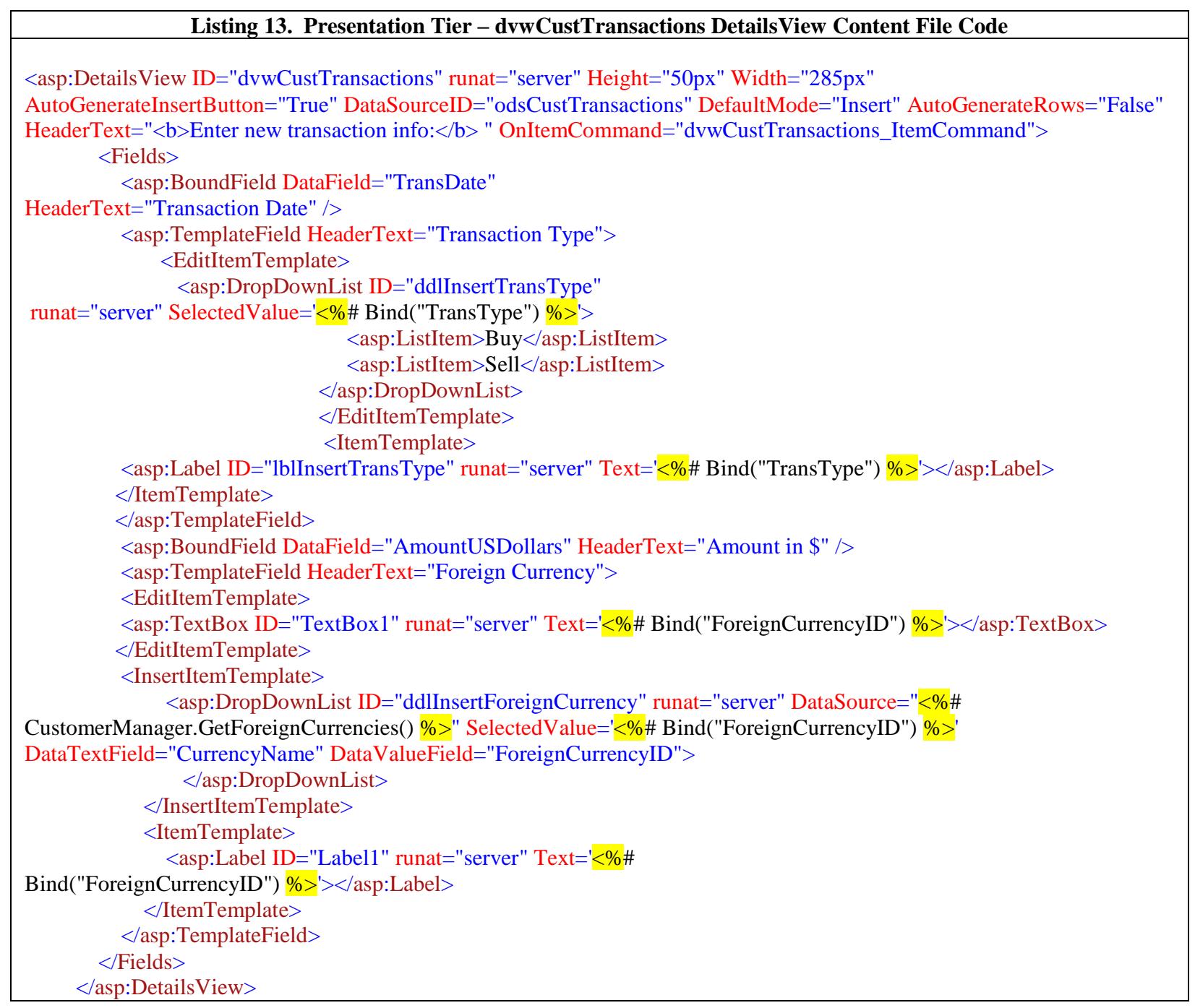

\section{CONCLUSION}

The growing popularity of data-driven Web applications has spurred the need for more integrated curricular materials that help instructors teach database and Web development concepts in a holistic manner. In this paper, we have presented application modules that help to fill this need. We encourage database and Web development instructors to adopt and extend the modules we have developed in an effort to better train the next generation of IS professionals.

\section{REFERENCES}

1. $\quad$ Celko, J., Joe Celko's SQL For Smarties: Advanced SQL Programming. Third Edition ed: Morgan Kaufmann.

2. Chen, P.P.-S., The Entity-Relationship Model - Toward a Unified View of Data. ACM Transactions on Database Systems, 1976. 1(1): p. 9-36. 1976

3. Codd, E.F., A Relational Model of Data for Large Shared Data Banks. Communications of the ACM 1970. 13(6). 1970 
4. Kung, M., Yang, S.C., and Zhang Y., The Changing Information Systems (IS) Curriculum: A Survey of Undergraduate Programs in the United States. Journal of Education for Business, 2006. 81(6): p. 291-300. 2006

5. Litecky, C., B. Prabhakar, and K. Arnett. The IT/IS Job Market: A Longitudinal Perspective. in SIGMIS Computer Personnel Research Conference. 2006. Claremont, CA: ACM.

6. Morien, R.I., A Critical Evaluation Database Textbooks, Curriculum and Educational Outcomes. Information Systems Education Journal, 2006. 4(44): p. 2-14. 2006

7. Patton, T. Determine when to use stored procedures vs. SQL in the code. 2005 [cited 2008 May, 28]; Available from: http://articles.techrepublic.com.com/5100-10878_11-5766837.html.

8. Prabhakar, B., C. Litecky, R., and K. Arnett, IT Skills in a Tough Job Market. Communications of the ACM, 2005. 48(10): p. 91-94. 2005

\section{NOTES}


NOTES 